\title{
On a Family of Random Noble Means Substitutions
}

\author{
M. MOLL* \\ Fakultät für Mathematik, Universität Bielefeld, Universitätsstraße 25, D-33615 Bielefeld, Germany \\ In 1989, Godrèche and Luck introduced the concept of local mixtures of primitive substitution rules along the \\ example of the well-known Fibonacci substitution and foreshadowed heuristic results on the topological entropy \\ and the spectral type of the diffraction measure of associated point sets. In this contribution, we present a \\ generalisation of this concept by regarding the so-called "noble means families", each consisting of finitely many \\ primitive substitution rules that individually all define the same two-sided discrete dynamical hull. We report \\ about results in the randomised case on topological entropy, ergodicity of the two-sided discrete hull, and the \\ spectral type of the diffraction measure of related point sets.
}

DOI: 10.12693/APhysPolA.126.539

PACS: $45.30 .+\mathrm{s}, 61.44 . \mathrm{Br}$

\section{The setting}

Consider the binary alphabet $\mathcal{A}_{2}=\{\mathrm{a}, \mathrm{b}\}$. For an arbitrary but fixed integer $m \geq 1$ and $0 \leq i \leq m$, we define the noble means substitution (NMS) rule $\zeta_{m, i}: \mathcal{A}_{2} \rightarrow \mathcal{A}_{2}^{*}$ by

$$
\zeta_{m, i}:\left\{\begin{array}{l}
\mathrm{a} \mapsto \mathrm{a}^{i} \mathrm{ba}^{m-i}, \\
\mathrm{~b} \mapsto \mathrm{a},
\end{array} \quad \text { where } \quad M_{m}:=\left(\begin{array}{cc}
m & 1 \\
1 & 0
\end{array}\right)\right.
$$

is its (unimodular) substitution matrix. We refer to $\zeta_{m}$ as a random noble means substitution (RNMS) where the special of the random Fibonacci substitution $(m=1)$ has first been studied by Godreche and Luck [1]. The family

$$
\mathcal{N}_{m}:=\left\{\zeta_{m, i} \mid m \in \mathbb{N}, \quad 0 \leq i \leq m\right\}
$$

is called a noble means family and each of its members is a primitive Pisot substitution with inflation multiplier $\lambda_{m}:=\left(m+\sqrt{m^{2}+4}\right) / 2$ and algebraic conjugate $\lambda_{m}^{\prime}:=\left(m-\sqrt{m^{2}+4}\right) / 2$. The two-sided discrete (symbolic) hull $\mathbb{X}_{m, i}$ of $\zeta_{m, i}$ is defined as the orbit closure of a fixed point in the local topology. Each $\mathbb{X}_{m, i}$ is reflection symmetric and aperiodic in the sense that it does not contain any periodic element. For fixed $m \in \mathbb{N}$, one observes that the $\zeta_{m, i}$ are pairwise conjugate and therefore all individual $\mathbb{X}_{m, i}$ coincide; see [2, Ch. 4] for background.

Now, we fix $m \in \mathbb{N}$ and a (strictly positive) probability vector $\boldsymbol{p}_{m}=\left(p_{0}, \ldots, p_{m}\right)$ and define a random substitution $\zeta_{m}$ on $\mathcal{A}_{2}$ by

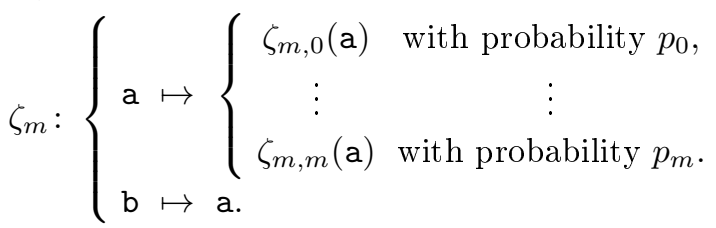

We refer to $\zeta_{m}$ as a random noble means substitution (RNMS). Both the substitution matrix and the inflation multiplier are the same as in the NMS case. We aim at

e-mail: mmoll@math.uni-bielefeld.de the local mixture of all members of $\mathcal{N}_{m}$, which means that we independently apply $\zeta_{m}$ to each letter of some word $w \in \mathcal{A}_{2}^{\mathbb{Z}}$. In this case, the two-sided discrete stochastic hull $\mathbb{X}_{m}$ is defined as the smallest closed and shiftinvariant subset of $\mathcal{A}_{2}^{\mathbb{Z}}$ with the property that $X_{m} \subset \mathbb{X}_{m}$, where

$$
\begin{gathered}
X_{m}:=\left\{w \in \mathcal{A}_{2}^{\mathbb{Z}} \mid w\right. \text { is an accumulation } \\
\text { point of } \left.\left(\zeta_{m}^{k}(\mathrm{a} \mid \mathrm{a})\right)_{k \in \mathbb{N}_{0}}\right\} .
\end{gathered}
$$

Both $\mathbb{X}_{m, i}$ and $\mathbb{X}_{m}$ are completely characterised by the legal subwords. Here, a word $w \in \mathcal{A}_{2}^{*}$ is $\zeta_{m}$-legal if there is a $k \in \mathbb{N}$ such that $w$ is a subword of at least one realisation of the random variable $\zeta_{m}^{k}(\mathrm{~b})$. The set of $\zeta_{m}$-legal words of length $\ell$ is henceforth denoted by $\mathcal{D}_{m, \ell}$. One can show that $\mathbb{X}_{m, i} \subsetneq \mathbb{X}_{m}$ by considering the subword bb and that the system $\left(\mathbb{X}_{m}, S\right)$, where $S$ denotes the shift, is topologically transitive but not minimal. Let us note that $\mathbb{X}_{m}$ is invariant under alterations of $\boldsymbol{p}_{m}$ as long as $\boldsymbol{p}_{m}$ is strictly positive.

\section{Topological entropy}

For $m \in \mathbb{N}$ and $n \geq 3$, the set of exact $R N M S$ words is given by

$$
\mathcal{G}_{m, n}:=\bigcup_{i=0}^{m} \prod_{j=0}^{m} \mathcal{G}_{m, n-1-\delta_{i j}},
$$

where $\mathcal{G}_{m, 1}:=\{\mathrm{b}\}, \mathcal{G}_{m, 2}:=\{\mathrm{a}\}$ and $\delta_{i j}$ denotes the Kronecker function. The product in Eq. (1) is understood via concatenation of words. Now, assume that $\boldsymbol{p}_{m}$ is strictly positive. The complexity function

$$
C_{m}: \mathbb{N} \rightarrow \mathbb{N}, \quad \ell \mapsto\left|\mathcal{D}_{m, \ell}\right|
$$

of $\zeta_{m}$ is unknown, but the knowledge of the exact RNMS words is enough [3] to compute the topological entropy $\mathcal{H}_{m}$ of $\zeta_{m}$ for any $m \in \mathbb{N}$ to be

$$
\begin{aligned}
\mathcal{H}_{m} & =\lim _{n \rightarrow \infty} \frac{\log \left(C_{m}\left(\ell_{m, n}\right)\right)}{\ell_{m, n}}=\lim _{n \rightarrow \infty} \frac{\log \left(\left|\mathcal{G}_{m, n}\right|\right)}{\ell_{m, n}} \\
& =\frac{\lambda_{m}-1}{1-\lambda_{m}^{\prime}} \sum_{i=2}^{\infty} \frac{\log (m(i-1)+1)}{\lambda_{m}^{i}}>0,
\end{aligned}
$$


where $\ell_{m, n}$ is the length of any word $w \in \mathcal{G}_{m, n}$. The numerical values of $\mathcal{H}_{m}$ for $1 \leq m \leq 4$ are shown in Table.

TABLE

Numerical values of $\mathcal{H}_{m}$ for $1 \leq m \leq 4$.

\begin{tabular}{c|c|c|c|c}
\hline \hline$m$ & 1 & 2 & 3 & 4 \\
\hline $\mathcal{H}_{m}$ & 0.44439 & 0.40855 & 0.37139 & 0.33862
\end{tabular}

One can prove that $\mathcal{H}_{m}>\mathcal{H}_{m+1}$ for all $m \in \mathbb{N}$ and $\mathcal{H}_{m} \stackrel{m \rightarrow \infty}{\longrightarrow} 0$.

\section{Ergodicity of $\left(\mathbb{X}_{m}, S\right)$}

The known concept of the induced substitution $[4$, Ch. 5] that acts on the alphabet of legal subwords of a fixed length can be generalised to the stochastic setting of the RNMS case. One obtains a random substitution rule $\left(\zeta_{m}\right)_{\ell}: \mathcal{D}_{m, \ell} \rightarrow \mathcal{D}_{m, \ell}^{*}$ and one can prove that the induced substitution matrix $M_{m, \ell}$ is a primitive matrix which enables the application of the Perron-Frobenius (PF) theory.

For fixed $m, \ell \in \mathbb{N}$, let $w \in \mathcal{D}_{m, \ell}$ be a $\zeta_{m}$-legal word. We define a shift-invariant probability measure $\mu_{m}$ on the cylinder sets $\mathcal{Z}_{k}(w)=\left\{v \in \mathbb{X}_{m} \mid v_{[k, k+\ell-1]}=w\right\}$ for any $k \in \mathbb{Z}$ by

$$
\mu_{m}\left(\mathcal{Z}_{k}(w)\right):=\boldsymbol{R}_{m, \ell}(w),
$$

where $\boldsymbol{R}_{m, \ell}(w)$ is the entry of the (statistically normalised) right $\mathrm{PF}$ eigenvector of $M_{m, \ell}$ according to the word $w$.

Theorem $1[5,6]$. Let $\mathbb{X}_{m} \subset \mathcal{A}_{2}^{\mathbb{Z}}$ be the hull of the random noble means substitution for $m \in \mathbb{N}$ and $\mu_{m}$ the shift-invariant probability measure of $E q$. (2) on $\mathbb{X}_{m}$. For any $f \in L^{1}\left(\mathbb{X}_{m}, \mu_{m}\right)$ and for an arbitrary but fixed $s \in \mathbb{Z}$

$$
\lim _{N \rightarrow \infty} \frac{1}{N} \sum_{i=s}^{N+s-1} f\left(S^{i} x\right)=\int_{\mathbb{X}_{m}} f \mathrm{~d} \mu_{m}
$$

holds for $\mu_{m}$-almost every $x \in \mathbb{X}_{m}$.

Theorem 1 implies that $\mu_{m}$ is ergodic. The proof can be accomplished via an application of Etemadi's formulation of the strong law of large numbers [7] and a suitable reorganisation of the summation over the characteristic function of some cylinder set.

\section{Cut and project}

The geometric realisations $\Lambda_{m, i}$ of fixed points of each noble means substitution can be derived as regular model sets within the cut and project scheme $\left(\mathbb{R}, \mathbb{R}, \mathcal{L}_{m}\right)$, where $\mathcal{L}_{m}:=\left\{\left(x, x^{\prime}\right) \mid x \in \mathbb{Z}\left[\lambda_{m}\right]\right\}$; see Fig. 1 . Here, the letters $\mathrm{a}$ and $\mathrm{b}$ are identified with closed intervals of length $\lambda_{m}$ and 1 , respectively, and the left endpoints are chosen as control points. The windows $W_{m, i}$ for $\Lambda_{m, i}$, in the generic cases $0<i<m$, are

$$
W_{m, i}:=i \tau_{m}+\left[\lambda_{m}^{\prime}, 1\right] \quad \text { with } \quad \tau_{m}:=-\frac{1}{m}\left(\lambda_{m}^{\prime}+1\right),
$$

while in the singular cases $i=0$ and $i=m$, one finds

$$
W_{m, 0}^{(\mathrm{a} \mid \mathrm{a})}:=\left[\lambda_{m}^{\prime}, 1\right), \quad W_{m, 0}^{(\mathrm{a} \mid \mathrm{b})}:=\left(\lambda_{m}^{\prime}, 1\right],
$$

$$
W_{m, m}^{(\mathrm{a} \mid \mathrm{a})}:=\left(-1,-\lambda_{m}^{\prime}\right], \quad W_{m, m}^{(\mathrm{b} \mid \mathrm{a})}:=\left[-1,-\lambda_{m}^{\prime}\right),
$$

distinguished according to the legal two-letter seeds. Now, one can prove that each member of the continuous RNMS hull $\mathbb{Y}_{m}$ is a relatively dense subset of an element of the LI class of the model set $\Theta\left(W_{m}\right)$, within the cut and project scheme of Fig. 1, with window $W_{m}=\left[\lambda_{m}^{\prime}-1,1-\lambda_{m}^{\prime}\right]$ and therefore a Meyer set by [8, Thm. 9.1]. The volume of the interval $W_{m}$ is minimal with this property, and it strictly contains $\bigcup_{i=0}^{m} W_{m, i}$; see Fig. 2 for an illustration in the case of $m=2$.

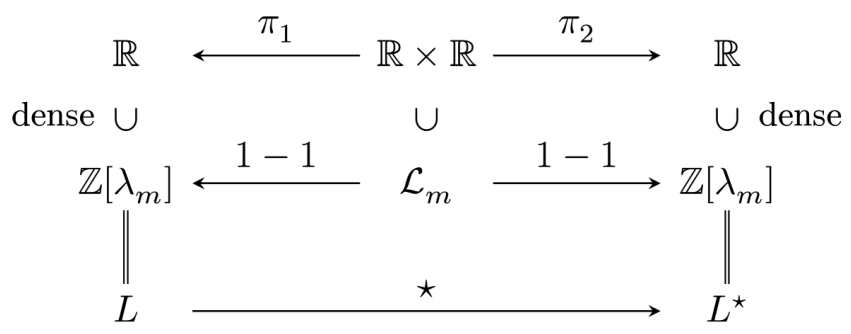

Fig. 1. Cut and project scheme for the noble means sets $\Lambda_{m, i}$.

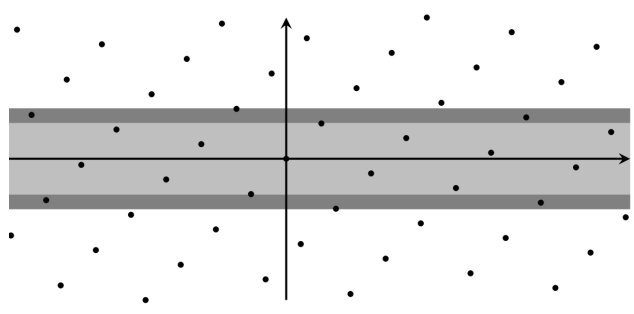

Fig. 2. The strip $\mathbb{R} \times \bigcup_{i=0}^{m} W_{m, i}$ (light) is strictly included in $\mathbb{R} \times W_{m}$ (dark). Here, this is illustrated for $m=2$.

Consequently, each geometric realisation of a random noble means word is a naturally arising instance of a Meyer set with entropy.

\section{Diffraction}

The diffraction of the NMS cases is well understood due to their characterisation as regular model sets [2, Ch. 9] whereas the results presented in [9, 10] suggest the presence of a continuous part in the diffraction spectrum in the RNMS case.

Because of Theorem 1, the suspension [11, 12] $\nu_{m}$ of $\mu_{m}$ on $\mathbb{Y}_{m}$ leads to a continuous and ergodic dynamical system $\left(\mathbb{Y}_{m}, \mathbb{R}, \nu_{m}\right)$. Now, let $\delta_{\Lambda}:=\sum_{x \in \Lambda} \delta_{x}$ be the Dirac comb for a random noble means set $\Lambda \in \mathbb{Y}_{m}$. One can compute the autocorrelation of $\delta_{\Lambda}$ to be $\nu_{m}$-almost surely given by

$$
\gamma:=\lim _{R \rightarrow \infty} \frac{\delta_{\Lambda_{R}} * \widetilde{\delta_{\Lambda_{R}}}}{\operatorname{vol}\left(B_{R}\right)}=\mathbb{E}\left(\delta_{\Lambda} \circledast \widetilde{\delta_{\Lambda}}\right),
$$

where $\Lambda_{R}:=\Lambda \cap B_{R}(0)$ and $\circledast$ denotes the volumeaveraged convolution by balls. The diffraction measure 
is given by the Fourier transform of $\gamma$ and reads

$$
\widehat{\gamma}=\lim _{R \rightarrow \infty} \frac{1}{\operatorname{vol}\left(B_{R}\right)}\left|\mathbb{E}\left(\widehat{\delta_{\Lambda_{R}}}\right)\right|^{2}+\lim _{R \rightarrow \infty} \frac{1}{\operatorname{vol}\left(B_{R}\right)} \mathbb{V}\left(\widehat{\delta_{\Lambda_{R}}}\right),
$$

where $\mathbb{E}$ and $\mathbb{V}$ refer to mean and variance with respect to the measure $\nu_{m}$. Now, the following two key properties finally lead to an explicit expression for $\widehat{\gamma}$.

- It is enough to study $\widehat{\gamma}$ on the basis of exact RNMS words, as defined in Eq. (1), because $\zeta_{m}$-legality of a word $w \in \mathcal{A}_{2}^{*}$ means that $w$ is a subword of a word in $\mathcal{G}_{m, n}$ for a suitably chosen $n \in \mathbb{N}$.

- It is not difficult to prove that

$$
\mathcal{G}_{m, n}=\left\{w \in \mathcal{A}_{2}^{*} \mid w=\zeta_{m}^{n-1}(\mathbf{b})\right\}
$$

and even more than the two stochastic processes, based on the substitution rule and the concatenation rule, are equal. Let us note that the equality in Eq. (3) means that there is at least one realisation of the random variable $\zeta_{m}^{n-1}(\mathrm{~b})$ that equals $w$.

For convenience, we restrict to $m=1$ in the following and define for $n \geq 2$ the complex-valued random variable $X_{n}(k)$ by

$$
X_{n}(k):= \begin{cases}X_{n-2}(k)+\mathrm{e}^{-2 \pi \mathrm{i} k \lambda_{1}^{n-2}} X_{n-1}(k), & \left\langle p_{0}\right\rangle, \\ X_{n-1}(k)+\mathrm{e}^{-2 \pi \mathrm{i} k \lambda_{1}^{n-1}} X_{n-2}(k), & \left\langle p_{1}\right\rangle,\end{cases}
$$

with $X_{0}(k)=\mathrm{e}^{-2 \pi \mathrm{i} k}$ and $X_{1}(k)=\mathrm{e}^{-2 \pi \mathrm{i} k \lambda_{1}}$. Here, $X_{n}(k)$ corresponds to exact RNMS words in $\mathcal{G}_{1, n+1}$. Therefore, we consider averaging over the sequence $L_{n}=$ $\lambda_{1}^{n}$ and find the following result.

Proposition 1 [4]. For any $n \in \mathbb{N}$, consider the function $\phi_{n}: \mathbb{R} \rightarrow \mathbb{R}_{+}$, defined by

$$
\phi_{n}(k):=\frac{1}{L_{n}} \mathbb{V}\left(X_{n}(k)\right) .
$$

The sequence $\left(\phi_{n}\right)_{n \in \mathbb{N}}$ converges uniformly to the continuous function $\phi: \mathbb{R} \rightarrow \mathbb{R}_{+}$, with

$$
\phi(k):=\frac{2 p_{0} p_{1} \lambda_{1}}{\sqrt{5}} \sum_{i=2}^{\infty} \lambda_{1}^{-i} \Psi_{i}(k) .
$$

Here, $\Psi_{n}: \mathbb{R} \rightarrow \mathbb{R}_{+}$is a bounded and smooth function that monotonically decreases in $n$, defined by

$$
\Psi_{n}(k):=\frac{1}{2}\left|\left(1-\mathrm{e}_{n-2}\right) \mathbb{E}_{n-1}-\left(1-\mathrm{e}_{n-1}\right) \mathbb{E}_{n-2}\right|^{2},
$$

where $\mathrm{e}_{n}:=\mathrm{e}^{-2 \pi \mathrm{i} k \lambda_{1}^{n}}$ and $\mathbb{E}_{n}:=\mathbb{E}\left(X_{n}(k)\right)$. This fixes the absolutely continuous part of $\widehat{\gamma}$. The pure point part can be computed via the recursion relation

$$
\mathbb{E}_{n}=\left(p_{1}+p_{0} \mathrm{e}_{n-2}\right) \mathbb{E}_{n-1}+\left(p_{0}+p_{1} \mathrm{e}_{n-1}\right) \mathbb{E}_{n-2},
$$

where $\mathbb{E}_{0}:=\mathrm{e}^{-2 \pi \mathrm{i} k}$ and $\mathbb{E}_{1}:=\mathrm{e}^{-2 \pi \mathrm{i} k \lambda_{1}}$. This yields

$$
\widehat{\gamma}(\{k\})=\lim _{n \rightarrow \infty} \frac{1}{L_{n}^{2}}\left|\mathbb{E}\left(X_{n}(k)\right)\right|^{2}
$$

and an approximation of $(\widehat{\gamma})_{\mathrm{pp}}$ and $(\widehat{\gamma})_{\text {ac }}$ is illustrated in Fig. 3 together with a sketch of the full diffraction, based on the recursion of Eq. (5) with $n=6$, in Fig. 4. Considering the Lebesgue decomposition $\widehat{\gamma}=(\widehat{\gamma})_{\mathrm{pp}}+(\widehat{\gamma})_{\mathrm{ac}}+$ $(\widehat{\gamma})_{\mathrm{sc}}$, we find that

$$
\widehat{\gamma}=(\widehat{\gamma})_{\mathrm{pp}}+\phi(k) \lambda,
$$

where $\lambda$ denotes the Lebesgue measure and $\phi$ - the density function of Eq. (4).

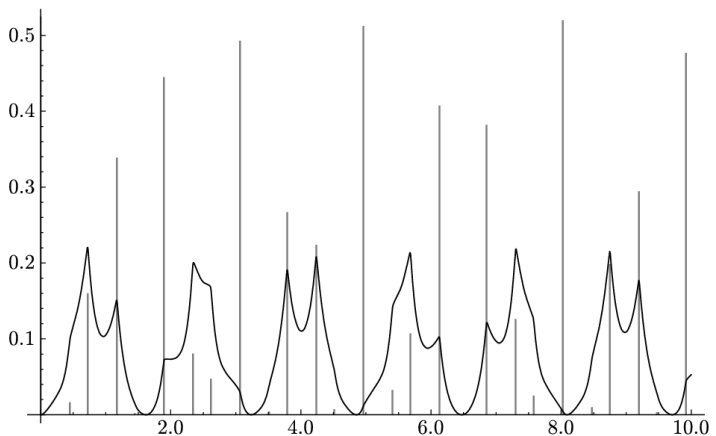

Fig. 3. The pure point (light) and absolutely continuous (dark) part of $\widehat{\gamma_{1}}$ with $\boldsymbol{p}_{1}=(1 / 2,1 / 2)$ is illustrated.

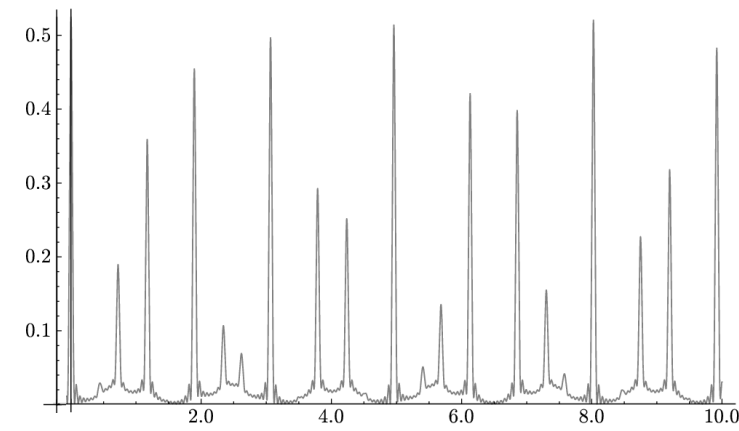

Fig. 4. Approximation of the diffraction measure for $m=1$ and with $\boldsymbol{p}_{1}=(1 / 2,1 / 2)$ is illustrated

It is possible to compute the pure point part from the recursion relation in Eq. (5). Another interesting approach comes from the theory of iterated function systems and inflation-invariant measures. Here, one finds that

$$
(\widehat{\gamma})_{\mathrm{pp}}=\sum_{k \in \mathcal{L}_{1}^{\circledast}}\left|\widehat{\eta}_{\mathrm{a}}\left(-k^{\prime}\right)+\widehat{\eta}_{\mathrm{b}}\left(-k^{\prime}\right)\right|^{2} \delta_{k},
$$

where $\mathcal{L}_{1}^{\circledast}=\pi_{1}\left(\mathcal{L}_{1}^{*}\right)=\mathbb{Z}\left[\lambda_{1}\right] / \sqrt{5}$, with $\mathcal{L}_{1}^{*}$ the dual lattice of $\mathcal{L}_{1}$, is the Fourier module. In the following, we write $\xi:=\lambda_{1}^{\prime}$. The invariant measures $\widehat{\eta_{\mathrm{a}}}, \widehat{\eta_{\mathrm{b}}}$ can be approximated via the recursion relation

$$
\left(\begin{array}{c}
\widehat{\eta_{\mathrm{a}}}(k) \\
\widehat{\eta_{\mathrm{b}}}(k)
\end{array}\right)=|\xi|^{n}\left(\prod_{\ell=1}^{n} p_{0} A_{\ell}(k)+p_{1} B_{\ell}(k)\right)\left(\begin{array}{c}
\widehat{\eta_{\mathrm{a}}}\left(k \xi^{n}\right) \\
\widehat{\eta_{\mathrm{b}}}\left(k \xi^{n}\right)
\end{array}\right),
$$

where the matrices $A_{\ell}(k)$ and $B_{\ell}(k)$ are given by

$$
\left(\begin{array}{cc}
\mathrm{e}^{-2 \pi \mathrm{i} k \xi^{\ell-1}} & 1 \\
1 & 0
\end{array}\right) \text { and }\left(\begin{array}{cc}
1 & 1 \\
\mathrm{e}^{-2 \pi \mathrm{i} k \xi^{\ell}} & 0
\end{array}\right)
$$

As $\xi^{n} \rightarrow 0$ for $n \rightarrow \infty$, an appropriate choice of the 
eigenvector $\left(\widehat{\eta_{\mathrm{a}}}(0), \widehat{\eta_{\mathrm{b}}}(0)\right)^{\mathrm{T}}$ for the equation

$$
\left(\begin{array}{ll}
1 & 1 \\
1 & 0
\end{array}\right)\left(\begin{array}{l}
\widehat{\eta_{\mathrm{a}}}(0) \\
\widehat{\eta_{\mathrm{b}}}(0)
\end{array}\right)=\lambda_{1}\left(\begin{array}{l}
\widehat{\eta_{\mathrm{a}}}(0) \\
\widehat{\eta_{\mathrm{b}}}(0)
\end{array}\right)
$$

fixes the recursion. Since $\widehat{\eta_{\mathrm{a}}}(0)+\widehat{\eta_{\mathrm{b}}}(0)$ must be the point density of some random golden means set, which always is $\lambda_{1} / \sqrt{5}$, one finds $\widehat{\eta_{\mathrm{a}}}(0)=1 / \sqrt{5}$ and $\widehat{\eta_{\mathrm{b}}}(0)=\left(\lambda_{1}-1\right) / \sqrt{5}$.

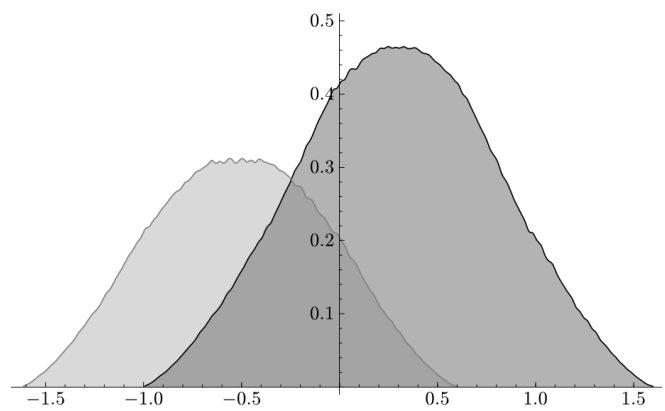

Fig. 5. Distribution of control points generated by a (dark) and $\mathrm{b}$ (light) in the internal space in the case of $\boldsymbol{p}_{1}=(1 / 2,1 / 2)$. The plot is generated by a lift of $\zeta_{1}^{32}(\mathrm{~b})$ (i.e. 2178309 points) to the internal space.

The distribution of control points in the internal space distinguished for a and $b$, respectively, is illustrated in Fig. 5.

\section{Acknowledgments}

The author wishes to thank Michael Baake, Tobias Jakobi and Johan Nilsson for helpful discussions. This work is supported by the German Research Foundation (DFG) via the Collaborative Research Centre (CRC 701) through the faculty of Mathematics, University of Bielefeld.

\section{References}

[1] C. Godrèche, J.M. Luck, J. Stat. Phys. 55, 1 (1989).

[2] M. Baake, U. Grimm, Aperiodic Order, Vol. 1, A Mathematical Invitation, Cambridge University Press, Cambridge 2013.

[3] J. Nilsson, Monatsh. Math. 166, 1 (2012).

[4] M. Queffélec, Substitution Dynamical Systems Spectral Analysis, 2nd ed., LNM 1294, Springer, Berlin 2010.

[5] M. Moll, Ph.D. Thesis, University of Bielefeld, 2013.

[6] M. Baake, M. Moll, in: Aperiodic Crystals, Eds. S. Schmid, R.L. Withers, R. Lifshitz, Springer, Dordrecht 2013, p. 19.

[7] N. Etemadi, Z. Wahrscheinlichkeitsth. verw. Geb. 55, 119 (1981).

[8] R.V. Moody, in: The Mathematics of Long-Range Aperiodic Order, Ed. R.V. Moody, NATO ASI Series C 489, Kluwer, Dordrecht 1997, p. 403.

[9] N. Strungaru, Can. J. Math. 65, 675 (2013).

[10] M. Baake, D. Lenz, C. Richard, Lett. Math. Phys. 82, 61 (2007).

[11] I.P. Cornfeld, S.V. Fomin, Y.G. Sinai, Ergodic Theory, Springer, New York 1982.

[12] M. Einsiedler, T. Ward, Ergodic Theory: With a View towards Number Theory, Springer, London (2011). 